\title{
Strategic Cost Management-A Review of Research Status at Home and Abroad
}

\author{
Yong Wang \\ School of Management, Jinan University, Guangzhou, China \\ Email:15521122513@163.com
}

How to cite this paper: Wang, Y. (2019) Strategic Cost Management-A Review of Research Status at Home and Abroad. Modern Economy, 10, 513-522. https://doi.org/10.4236/me.2019.102035

Received: January 18, 2019

Accepted: February 16, 2019

Published: February 19, 2019

Copyright $\odot 2019$ by author(s) and Scientific Research Publishing Inc. This work is licensed under the Creative Commons Attribution International License (CC BY 4.0).

http://creativecommons.org/licenses/by/4.0/

\begin{abstract}
Cost management is a long-standing research topic in the field of management accounting. In today's, cost management has been closely linked to the company's strategy, and has become an important way for enterprises to build competitive advantage and create core competitiveness. In order to understand the development of strategic cost management in recent years, in order to inspire future research directions, this paper mainly selects the domestic and international literature on strategic cost management issues from 2013 to 2017, their research content, research framework, and research methods. The conclusions of the study are summarized. It is found that the strategic cost management research shows the characteristics of using empirical research and clear research framework. Finally, based on this, the future research is proposed.
\end{abstract}

\section{Keywords}

Strategic Cost Management, Research Status, Literature Review

\section{Introduction}

Cost management is a long-standing research topic in the field of management accounting. Today, cost management has been closely linked to the company's strategy, and has become an important way for enterprises to build competitive advantage and create core competitiveness. The definition of strategic cost management is to identify cost drivers from the source of cost from a strategic perspective, and to manage costs in the value chain, that is, to use cost data and information to provide strategic cost information for each critical step of strategic management. In order to facilitate the formation of competitive advantage and the creation of core competitiveness, traditional cost management focuses on cost-cutting technologies and methods, while strategic cost management is not 
based on cost-cutting. It focuses on getting as much use value as possible with as little cost as possible. Strategic cost management is guided by cost-effectiveness. If it can enhance the competitive advantage of enterprises, it is in line with the spirit of strategic cost management to increase costs appropriately. Compared with foreign countries, China's strategic cost management research started late. This paper attempts to make a brief review of the relevant literature on strategic cost management in the past five years at home and abroad, and summarizes the research results, hoping to provide some inspiration for future research.

This literature review is mainly divided into the following parts: First, a brief statistical analysis of the selected literature, including research content, research methods, source journals, etc.; secondly, according to different research contents, the study of strategic cost management issues. The results are sorted out and summarized; finally, on this basis, some ideas provided for future research directions.

\section{Literature Selection and Related Analysis}

\subsection{Literature Selection}

This paper selects foreign and domestic literature on strategic cost management, with a time span of 2013 to 2017. Among them, the foreign literature comes from the database EBSCO-Academic Search Premier (including more than 1500 full-text journals of SSCI), using the keyword "strategic cost management" to search, and eliminating the literature with low degree of "strategic cost management" theme. Finded 12 foreign literatures. Domestic literature comes from China national knowledge internet. In the last six years, there were 24 papers on the theme of strategic cost management, including 6 in 2013, 7 in 2014, 1 in 2015, 8 in 2016, and 2 in 2017. From this we can also see that although strategic cost management is an important research topic, it has not been studied much in recent years.

\subsection{Related Analysis}

Among the 24 papers, the research on strategic cost management mainly focuses on the fields of performance evaluation and cost motivation, and the research methods used include empirical research, field research and analytical research. See Table 1 for details:

It can be seen that in recent years, the most commonly used method for strategic cost management is empirical research, followed by field research and analytical research. To a certain extent, this shows that the research on strategic cost management in 2013-2017 is mostly based on the data obtained and relatively standardized. There is not much difference between domestic and foreign research methods.

Research on strategic cost management generally does not exist independently of the value chain (e.g. Kaplan and Norton, 1996, 2004). In the practice of strategic cost management, the use of differential analysis, behavior analysis and standard cost methods to analyze cost data, find ways to improve the cost to 
Table 1. Distribution of research methods (2013-2017).

\begin{tabular}{cccc}
\hline & Empirical Research & Field Research & Analytical Research \\
\hline Number of articles & 16 & 6 & 2 \\
Proportion & $66.67 \%$ & $25 \%$ & $8.33 \%$ \\
\hline
\end{tabular}

improve cost performance is called executive cost management; and use organizational design, product design and process. The way in which tools such as design establish a cost structure that is aligned with corporate strategy is called structural cost management. The study of strategic cost management is also divided into two categories. One is to study whether and how companies use accounting data for Porter's value chain analysis, such as Hergelt and Morris (1989, SMJ), Shank (1989, JMAR), Shank and Govindarajan (1992, JMAR). The other is to study the relationship between corporate strategy and cost structure, as well as the causal relationship between the level of operations and the resources required (i.e. cost drivers), such as Banker and Johnston (1993, TAR), Ittner et al. (1997, JMAR), Maher and Marais (1998, JAR). Anderson (2007) summarizes the research results in the field of strategic cost management and proposes a strategic and strategic cost management chart. In this framework, the central issues of strategic cost management are value chain analysis, cost driver analysis, and activity-based costing (Lord, 1996, MAR; Banker and Johnston, 2007). Among them, accounting scholars have the most extensive research on cost drivers (e.g. MacArthur and Stranahan, 1998, JMAR).

\subsection{Research Content}

Since the discussion and analysis of cost management can only be carried out under specific environmental settings, most of the research on strategic cost management is based on specific industries, such as air transport, manufacturing, and medical industries. Similarly, in the 24 selected domestic and foreign literatures, the research content is mostly concerned with the strategic cost management issues of these industries. The details will be described in detail in the third section.

\section{Literature Review}

\subsection{Research on Strategic Cost Management in Air Transportation Industry}

Traditional cost accounting systems rely on capacity-based metrics such as production, direct labor hours, and machine hours for costing and allocation. The cost of such products is often biased or even wrong, causing managers to make mistakes. Short-term business decisions, such as whether to continue production or stop production of a product, whether to continue to supply. The wrong cost system can even have significant adverse consequences for the company's budget management, variance analysis and accountability accounting system. Therefore, 
companies need more accurate cost measurement and begin to implement the operating cost system. Cooper and Kaplan's field research found that managers have begun to focus on operational metrics through interviews with several corporate executives, but their research is still lacking systematic empirical evidence [1]. Foster and Gupta (JAE) studied the manufacturing costs of a company's 37 workshops and refined several operational metrics based on the company's manufacturing process. Although they conducted empirical analysis on manufacturing costs and operational indicators, they did not find empirical evidence of a significant relationship between the two.

Banker and Johnston (TAR) is an empirical paper on the strategic cost management of the US air transport industry. Based on 28 US airlines, the author examines the empirical relationship between resource consumption and capacity-based cost drivers and operational cost drivers in the air transport industry, and explains the management value of operational cost drivers. The author's research shows that the cost drivers of operations are significantly related to resource consumption, and the cost drivers of operations reflect the different competitive strategies of airlines.

The difficulty and focus of empirical research in the field of strategic cost management is usually variable selection. Because unlike other accounting branches, the definition of variables in the field of management accounting is very difficult. The measurement of variables lacks a unified and accepted standard. Researchers need to make appropriate screening and selection according to the specific problems of the research and the value chain of the company.

In the past, the literature believes that in the air transport industry, total passenger and cargo mileage is a cost-based cost driver (Sickles, JE; Sickles and Good, JE), but the author believes that the total passenger and cargo miles are only suitable for dealing directly with passengers and goods. Most of the other costs are very low. For example, fuel consumption and flight service operations are more related to aircraft size, seating capacity, flight distance, and more. Therefore, the author proposes that the effective seat mileage and effective cargo mileage should be used as the capacity-based cost drivers, and the two should be transformed and combined according to the research needs, and finally form the most important production cost driver of the paper: the capacity seat mileage.

According to the rules of Hayes and Clark (Interfaces) and Miller and Vollman (HBR) in the manufacturing industry, the operational cost drivers of the air transport industry are determined with reference to manufacturing product quality, product line diversity, manufacturing processes and production batches. Such as aircraft model, flight density, aircraft shift control, etc. The author's analysis pointed out that since the United States canceled air traffic control, each airline adopted a different competition strategy, mainly reflected in the establishment and use of the hub center operating system. The establishment of a hub center allows airlines to use more aircraft to transport more passengers at a time, thereby reducing costs, but at the same time increasing the company's management costs (because the aircraft will spend more time on the ground). Therefore, 
hub concentration and hub coverage are also operational cost drivers selected by the author.

\subsection{Strategic Cost Management Research in the Medical Industry}

$\mathrm{Xu}$ Yuanyuan (2014) studied the relationship between macro-public health care reform and micro-health care institutions' response measures, and published it in accounting research. Xu Yuanyuan's research shows that medical reform is a macroscopic and complex project, and it is necessary to constantly adjust various policies to explore the path of medical reform that suits China's national conditions. Xu Yuanyuan's research focused on the medical reforms aimed at breaking the "medical treatment by medicine", starting from the perspective of the interaction between the design of top-level medical reform system and micro-medical services. By eliminating the clue of the drug addiction policy to the reconstruction of the pharmaceutical supply value chain, the macro-reform exploration and micro-public hospital response measures are linked together, and the strategic cost management theory is used as a guide to analyze how public hospitals respond to macro-medical reform requirements and in the reconstructed medical supply value chain environment, clear new strategic positioning, discuss the corresponding public hospital compensation, and then make recommendations on the public hospital cost driver identification, control and strategic cost management performance evaluation.

$\mathrm{Xu}$ Yuanyuan's research has led to the exploration of adjustments to regular financial subsidies and incentive financial subsidies for public hospitals. According to the commonality of public hospitals, the basic medical treatment projects will be set up with a loss and no subsidy allowance, and regular hearings will be carried out to update and optimize the subsidy projects. Differentiated subsidies will be implemented for the special circumstances of different types of hospitals, and the financial subsidies and the number of services provided will be provided. The quality and related financial information are linked, and a new performance appraisal subsidy system is established. The assessment results are based on the assessment weights of the assessment indicators such as the number of visits, the average cost control rate, the drug control ratio, the patient satisfaction rate, and the medical service cost. Subsidy basis encourages public hospitals to improve the quality of medical technology and services and better serve patients.

The smooth progress of "public welfare" medical reform requires multiple measures to protect it, and the elimination of drug addiction and compensation mechanism design is only one of them. This requires the active coordination and support of the micro-subjects, a full understanding of the original intentions of the reform and policy limitations, adjustment of its strategic positioning in the reform, good feedback on policy implementation and suggestions for further policy recommendations, and more important is to do well. The basic cost management of micro-subjects, improves the quality of medical services, enhances the trust of the pharmaceutical supply chain, and helps medical reform 
exploration.

\subsection{Research on Strategic Cost Management in Manufacturing Industry}

Oyewo Babajide Michael (2013)'s study of Nigeria found that the 2008 financial crisis caused business priorities to shift from growth rates and profits to survival issues until the economy improved. One thing that can be understood is the emphasis on cost; therefore, cost management is seen as one of the best ways to maintain profitability in the face of softening sales and shrinking profits. The purpose of his research was to determine whether Nigerian companies actually use strategic cost management (SCM) technology, and the extent of their use (especially in the Nigerian manufacturing and financial services industries) to identify factors that influence the adoption of strategic cost management, and whether the strategy can be used. Cost management is used as a survival competitive strategy during the recession. Statistical procedures were performed on the collected data using the Mann-Whitney test. The study found that although Nigerian companies accept the philosophy of strategic cost management (SCM), there are challenges in the Nigerian environment that hinder their adoption and implementation. Manufacturing focus also leverages strategic cost management (SCM) tools compared to financial services companies. Nigerian companies are encouraged to adopt strategic cost management tools, and the Nigerian government has developed policies to create an enabling environment for the adoption of strategic cost management (SCM).

Oyewo Babajide Michael's research investigates how to use strategic cost management (SCM) in the manufacturing and financial services industries as a competitive tool for corporate survival during recession [2]. Although global financing has a negative impact on global companies, the impact is not entirely negative. The recession has forced companies to focus on developing cost management strategies to ensure their survival and continued relevance in the business environment. It also forces them to adopt and adapt to existing strategic cost management (SCM) technologies. The study also found that strategic cost management (SCM) technologies are feasible, applicable, and implementable in the Nigerian environment: but they are more widely used in Nigerian manufacturing than in the financial services industry.

\subsection{Research of Other Aspects}

Ye Kangtao, Zhang Wei, and Zhang Yixin (2014) Research on the relationship between corporate strategy differences and accounting information value shows that when the corporate strategy deviates from the industry's conventional strategy, the value correlation of the owner's equity is higher, and the value correlation of net profit is the lower. After controlling the impact of corporate-level characteristics such as corporate bankruptcy risk, earnings persistence, income growth, firm maturity and stock liquidity, the research conclusion remains unchanged (this indicates that investors are also concerned about Accounting and 
strategic information in stock pricing process). Moreover, investors' understanding of accounting information relies on strategic information. Therefore, increasing the disclosure of strategic information helps investors better understand financial statements, and their research helps to improve the company's information disclosure system. And better understand the value relevance of accounting information. Xie Dongming and Wang Ping (2013) studied the relationship between ecological economic development model and corporate environmental cost strategic control, and the meaning, characteristics and driving theory of corporate environmental cost strategic control. They expounded the mechanism of strategic control of corporate environmental cost under the model of ecological economic development, and demonstrated that the modern mode based on "strategic cost control" is an optimal environmental cost control model, which can make the total environmental cost equal to the control cost. The damage cost is zero, the total environmental cost is the lowest, and the zero emission is basically realized. Finally, the product life cycle cost management, operation cost management, material flow cost accounting-quantitative analysis of resource loss and incentive evaluation of implementing environmental cost strategic control is introduced. The implementation and application of four methods, such as mechanism, in enterprise environmental cost strategy control. Xie Dongming and Wang Ping's research laid a certain theoretical foundation for the implementation of environmental cost control in China's enterprises under the ecological economic development model, and provided specific implementation strategies and methods for enterprises to build an ecological economic development model to achieve economic benefits. The win-win situation with environmental benefits has an important driving role, and it has more positive and practical significance for accelerating China's "ecological civilization construction" and creating a "blue China" grand blueprint. Lapsley I and Rekers J V. (2017, MAR) studied the relationship between accounting and popular culture [3]. Specifically, by studying the novel setting of the music world, we have increased our understanding of the potential of strategic management accounting. Using a case study approach, this study challenges traditional thinking from the perspective of "strategic practice" using the process of developing popular theater portfolio activities. The results of the study show that strategy is a complex practice and an intrinsic social process: the drama producers negotiate a market path by verifying the involvement of intermediaries, contributing to and disseminating the reputation of new cultural products, thereby supporting the strategic process. Research by Khodzytska V (2014, AF) shows that control is a tool for collecting, analyzing and evaluating the necessary information about the company's condition [4]. Managers need this information to make effective management decisions that ultimately reduce risk levels. This paper is devoted to the nature and aspects of strategic control implementation as a strategic cost management tool for companies. Focus on the main aspects of strategic control impact making effective decisions on strategic cost management. It reveals the differences between operational and strategic controls and provides the vision of 
the company's strategic control structure. Liu Q. (2014) shows that strategic cost management is the cornerstone of successful implementation of strategic management, and applying innovative strategic methods will help modern enterprises to seize cost management [5]. As for the wide distribution of modern enterprise departments, the cost management issues are complex and diverse. Therefore, in strategic cost management, modern enterprises cannot simply copy other business practices; they should be operated in the company in conjunction with the specific requirements of strategic cost control [6]. In response to the specific problems of cost management, effective measures are taken to continuously improve the implementation of the business strategy of cost management, and ultimately promote the long-term prosperity and development of modern enterprises.

\section{Conclusion}

By reviewing and sorting out the selected literatures on domestic and international strategic cost management from 2013 to 2017, we can find the following characteristics: First, from the perspective of the research methods used, there are mainly empirical research, field research and analytical. Three research methods are studied, and the most used ones are empirical research and field research, and the objectivity is stronger. Second, from the theory involved, in addition to the theory commonly used in economics, the relevant theories of sociology, psychology, and organizational behavior are often used, and the cross-disciplinary characteristics are obvious. In addition, the theoretical sources of foreign strategic cost management literature are relatively rich, and the theoretical basis is relatively solid. In contrast, the relevant domestic literature in China appears to be somewhat weak and not profound enough. Third, from the research content, the research on strategic cost management of specific industries still occupies a major part. Some scholars have studied strategic cost management from different perspectives such as accounting information value relevance, ecological economic development, and popular culture. Compared with foreign research, in recent years, China's research on strategic cost management has also developed rapidly, and the research results are rich.

\section{Shortcomings}

The content of the article is relatively simple, and the previous literature needs to be more deeply combed and grasped. The collection of documents should be more comprehensive. Mastering a comprehensive and large amount of literature is a prerequisite for writing a good review. Otherwise, it is impossible to write a lot of reviews by simply collecting a little information, and even writing articles does not become a summary at all. Note the representativeness, reliability, and scientific nature of the cited literature. There may be similar views in the collected literature, and some literatures have differences in reliability. Therefore, when citing documents, attention should be paid to the selection of documents 
with good representativeness, reliability.

\section{Research Outlook}

\subsection{The Significance of Foreign Research for China}

Foreign research in this area has the following two points worthy of our reference: First, strengthen the theoretical foundation. If the research based on data analysis is more reliable, the solid theoretical foundation is the icing on the cake, which will make the research more logical and improve the ideological nature of the article [7]. Second, innovative research perspective. The domestic strategic cost management literature only focuses on the exploration of different industries, and rarely touches other aspects [8]. This requires domestic scholars to be more adept at discovering problems in practice, and to conduct more extensive and in-depth research on the problems of strategic cost management in practice in light of China's national conditions.

\subsection{Prospects for Future Research}

In recent years, on the theme of strategic cost management, domestic and foreign scholars have paid great attention to their research in specific environments. Although a lot of conclusions have been drawn in this respect, the direction of influence on some factors is not conclusive [9]. Moreover, it can be considered whether the determined influencing factors will still play a role in strategic cost management when the environment changes, that is, to explore the robustness of strategic cost management.

In addition, compared with previous years, the application of field research is increasing. China's literature on strategic cost management has also begun to use field research methods to solve problems [10]. Therefore, it is expected that the development of field research will be more rapid in the future. The research on strategic cost management and even the field of management accounting pay more attention to the role of people in it [11]. The characteristics of interdisciplinary are more and more obvious, and it is necessary to contact experimental economics and society [12]. Therefore, we need to have a broader perspective.

\section{Conflicts of Interest}

The author declares no conflicts of interest regarding the publication of this paper.

\section{References}

[1] Rao, U.L. (2016) Iot as a Stategic Cost Management Tool. The MA Journal, 15.

[2] Koo, K. (2015) Latent Talent of Generalist CEOs on Strategic Cost Management. Working Paper.

[3] Lapsley, I. and Rekers, J.V. (2017) The Relevance of Strategic Management Accounting to Popular Culture: The World of West End Musicals. Management Accounting Research, 35, 47-55. https://doi.org/10.1016/j.mar.2017.01.001 
[4] Grant, R.M. (2016) Contemporary Strategy Analysis: Text and Cases Edition. John Wiley \& Sons, Hoboken.

[5] Guenther, T.W. and Gaebler, S. (2014) Antecedents of the Adoption and Success of Strategic Cost Management Methods: A Meta-Analytic Investigation. Journal of Business Economics, 84, 145-190. https://doi.org/10.1007/s11573-013-0695-Z

[6] Liu, Q. (2015) Research on Strategic Cost Management in Modern Enterprises. Management \& Engineering, 21, 1838-5745.

[7] Banerjee, B. (2014) Strategic Cost Management: Conceptual Underpinning. $M A$ Journal.

[8] Savić, B., Vasiljević, Z. and Đorđević, D. (2014) Strategic Cost Management as Instrument for Improving Competitiveness of Agribusiness Complex. Economics of Agriculture, 61, 1005-1020.

[9] Michael, O.B. (2013) Strategic Cost Management as a Recession Survival Tool in the Nigerian Manufacturing and Financial Service Industries.

[10] Khodzytska, V. (2014) Strategic Controlling as Methodological Tool for Strategic Management of Expenses. Accounting and Finance, 4, 146-153.

[11] Anderson, S.W. (2007) Managing Costs and Cost Structure throughout the Value Chain: Research on Strategic Cost Management. Handbook of Management Accounting Research, 2, 481-506.

[12] Ferreia, A. and Otley, D.T. (2009) The Design and Use of Performance Management Systems: An Extended Framework for Analysis. Management Accounting Research, 20, 263-282. https://doi.org/10.1016/j.mar.2009.07.003 